\title{
Concreto Polimérico Reforzado con Fibras de Luffa
}

\author{
Gonzalo Martínez-Barrera, Miguel Martínez-López, Elisa Martínez-Cruz \\ Laboratorio de Investigación y Desarrollo de Materiales Avanzados (LIDMA), Facultad de Química, \\ Universidad Autónoma del Estado de México, Km.12 de la carretera Toluca-Atlacomulco, \\ CP 50200, San Cayetano-México (e-mail: gonzomartinez02@yahoo.com.mx).
}

Recibido Dic. 21, 2012; Aceptado Feb. 12, 2013; Versión final recibida Abr. 06, 2013

\begin{abstract}
Resumen
Se elaboró un tipo de concreto polimérico añadiendo fibras de luffa con el fin de mejorar el la resistencia a la compresión y a la flexión, así como el grado de elasticidad. Se prepararon probetas de concreto polimérico con $30 \%$ en volumen de resina poliéster y el $70 \%$ restante con arena sílice y diversas concentraciones de fibras de luffa $(0.3,0.6$ y $0.9 \%$ en volumen), así como pobretas testigo sin fibra. Las probetas testigo, sin fibra, fueron sometidas a radiación gamma. La evaluación de la resistencia mecánica a la compresión y a la flexión de las probetas de concreto polimérico se realizó en una maquina universal de pruebas mientras que el módulo de elasticidad dinámico se determinó con un equipo de ultrasonido. Una vez efectuado las pruebas mecánicas a los concretos, se analizaron regiones de las fibras de luffa en un microscopio electrónico de barrido. Los resultados muestran disminución en los valores de la resistencia a compresión y la flexión y la elasticidad para las probetas con fibras. Las propiedades mejoraron para las probetas irradiadas.
\end{abstract}

Palabras clave: Concreto polimérico, fibras de luffa, radiación gamma, propiedades mecánicas.

\section{Polymer Concrete Reinforced with Luffa Fiber}

\begin{abstract}
A polymer concrete was prepared adding luffa fiber with the aim of improving compressive and flexural strength as well as elasticity. Polymer concrete specimens were prepared with unsaturated polyester resin with $30 \%$ in volume being the remaining $70 \%$ siliceous sand and luffa fibers at several concentrations $(0.3$, 0.6 and 0.9 vol\%). Specimens without fibers were evaluated and gamma irradiating. The evaluation of the mechanical resistance to compression and flexion of the specimens were done in a universal testing machine while elasticity modulus was determined with ultrasound equipment. After the mechanical tests the luffa fiber areas were analyzed using a scanning electronic microscopy. The results show that compressive strength, flexural strength and elasticity decrease their values for the specimens with fibers. The properties improved for the irradiated specimens.
\end{abstract}

Keywords: polymer concrete, luffa fibers, gamma radiation, mechanical properties. 


\section{INTRODUCCIÓN}

De los materiales de construcción existentes, el concreto hidráulico a base de cemento Portland (CCP), es el material más utilizado para construcciones en el mundo. No obstante, presenta algunas desventajas como el grado de porosidad que afecta directamente su rendimiento y limitan sus aplicaciones; la débil adherencia a ciertos materiales; baja resistencia a medios agresivos y al agua salada; así como pobre aislamiento eléctrico. Razones por las cuales se han buscado y propuesto tecnologías alternas que toman en cuenta otros materiales, como los polímeros. Los concretos modificados con polímeros han mostrado mejoramiento en las propiedades mecánicas de resistencia a la compresión y a la flexión en comparación con concretos hidráulicos sin polímeros.

El Concreto Polimérico (CP) es un material compuesto que consta de la mezcla de una resina termoestable y agregados minerales, a diferencia del agua y el cemento tipo Portland utilizados en la elaboración del cemento hidráulico. Las propiedades mecánicas del concreto polimérico dependen de las propiedades físicas y químicas tanto de la resina como de los agregados minerales (Mehdi A., 2011). El concreto polimérico es de tres a cinco veces más resistente que el concreto hidráulico (Martínez-Barrera, 2009), pero su fragilidad en el punto de falla ha limitado su utilidad en aplicaciones donde intervienen cargas pesadas; siendo necesario mejorar la dureza y el comportamiento esfuerzo-deformación, primordialmente en lo referente al módulo de Young. Para solucionar este problema se han propuesto: a) llevar a cabo un proceso de post-curado, consistente en calentar a cierta temperatura el concreto por determinado tiempo, realizándolo por etapas, o bien b) reforzarlo con fibras, entre las que se encuentran fibras sintéticas, naturales o de carbono; esta ultimas influyen notablemente en el concreto, mejorando su capacidad y modificando su comportamiento estructural en cuanto a rigidez y ductilidad (Valcuende M. et al., 2004). El reforzamiento con fibras en concreto hidráulico se ha venido realizado desde los años 60 's, conociéndose con el nombre de Fiber Reinforced Concrete (FRC), se ha encontrado que la fibras añadidas al concreto mejoran la resistencia a la tracción, a la fatiga, al impacto y a la abrasión; se tiene mayor control con la contracción y con la estabilidad térmica (Zollo R.F.,1997).

No obstante los esfuerzos realizados en el área de los concretos reforzados con fibras (FRC), sólo algunos grupos de investigación han abordado la tarea de resolver la problemática del reforzamiento de concreto polimérico, y en consecuencia la literatura es escasa. Se ha encontrado que la adición de fibras poliméricas sintéticas refuerza el carácter elastomérico de la matriz polimérica, por lo general una resina polimérica termoestable, como las resinas epoxi o las de poliéster insaturado; además de mejorar la ductilidad y disminuir el módulo de elasticidad (Martínez-Barrera y Brostow, 2009).

El uso de materia prima natural como las fibras naturales, entre ellas las de Luffa, pueden ser una excelente alternativa para el desarrollo de concretos poliméricos reforzados más amigables al medio ambiente y que cumplan con las demandas de autoridades a nivel mundial y que atiendan la sustentabilidad global. Los pocos estudios de concreto polimérico reforzado con fibras naturales, contemplan el uso de fibras de piassava para disminuir el peso del concreto, mejorar el comportamiento en flexión, así como disminuir el valor al que se logra falla frágil (Reis J.M.L. y Carneiro E.P., 2013); también se han utilizado fibras de bambú para mejorar la propiedades de tracción y la adhesión entre el agregado mineral y la resina polimérica, en base al poder antioxidante del bambú (Santos Delgado P. et al., 2012). Por otro lado, los grupos polares en la fibra natural de sisal interactúan físicamente con la resina poliéster y retardan la reacción de curado, lo cual puede permitir la manipulación durante la elaboración del concreto polimérico, el cual en promedio polimeriza en dos horas (Pistor V. et al., 2012).

Las fibras de luffa, también conocidas como esponjas, se utilizan con frecuencia para la ducha diaria y el lavado de utensilios. La información sobre su uso en la elaboración de compositos es muy escasa, y mucho menos de los efectos que le puede causar la radiación gamma, esto debido primordialmente a ser un material de estructura química compleja (Ghali et al., 2009; Oboh y Aluyor, 2009; Tanobe et al., 2005); la cual consiste principalmente de celulosa (54\%), hemi-celulosa (20\%) y lignina (15\%). La estructura de la luffa es de carácter fibroso, tiene una región central y capas externas en la cual las fibras están colocadas en arreglos multidireccionales formando una red, con la presencia de micro-canales y poros con diámetros de 10-20 $\mu \mathrm{m}$. Características que pueden ser usadas como ventajas en la elaboración de materiales compuestos, abriendo un abanico de oportunidades de investigación (Altinisik A. et al., 2010; Boynard C., 2010).

En el concreto polimérico sólo acontecen interacciones del tipo físico entre las fibras y la resina poliéster; situación que podría cambiar con el uso de la radiación gamma, con la cual se puede modificar la estructura química y la morfología tanto de la resina como de las fibras de luffa, y con esto lograr un mayor grado de compatibilidad (Martínez-Barrera et al., 2008). Cabe mencionar, que el grado de radiactividad y los índices de riesgo se ha podido medir en algunos materiales de construcción, como las pizarras (Miro C. et al., 2010). 
La radiación gamma causa en los polímeros tres diferentes procesos: rompimiento o entrecruzamiento de cadenas, e injerto. La permanencia de alguno de estos procesos depende de la estructura química del polímero y de la dosis aplicada (Clough, 2001). Comparado con los procesos térmicos o por ataque químico, la radiación gamma tiene las siguientes ventajas: a) la iniciación de la radiación no requiere de ninguna energía de activación; b) no se requiere de catalizadores; c) la iniciación es homogénea; d) el proceso se puede llevar a cabo a cualquier temperatura y puede ser interrumpido a un tiempo de reacción especifico; e) la reacción de terminación es prácticamente controlada y f) la temperatura durante la reacción de inicialización es mantenida (Cruz-Zaragoza y Martínez-Barrera, 2009). Mediante el uso de radiación es posible obtener películas poliméricas conteniendo silanos órgano funcionales, las cuales permiten controlar la pasivación de substratos y en consecuencia mejoran la resistencia a la corrosión; evitando el uso de sustancias con un elevado grado de toxicidad, como el cromo hexavalente que regularmente se utiliza para tal aplicación (Kunst S. et al., 2011).

En el presente trabajo se estudia el efecto de las fibras de Luffa y la radiación gamma en las propiedades mecánicas del concreto polimérico elaborado con arena sílice y resina poliéster insaturada.

\section{MATERIALES Y MÉTODOS}

La resina de poliéster insaturada isoftálica fue donada por la empresa Matexplás (Porto Portugal), comercializada con el nombre A AROPOL IS 4633. La arena sílice utilizada es comercializada con el nombre SP55, con una densidad de $2.6 \mathrm{~g} / \mathrm{cm}^{3}$ y diámetro promedio de $250 \mu \mathrm{m}$ (malla 60); las fibras de luffa tienen un diámetro entre 150-200 $\mu \mathrm{m}$ con capacidad de absorción de agua de $13.6 \mathrm{~g} / \mathrm{g}$ y área superficial de $123 \mathrm{~m}^{2} / \mathrm{g}$.

\section{Elaboración de Concretos Poliméricos}

Las probetas de concreto polimérico se elaboraron con 30\% de resina poliéster, y el $70 \%$ restante con arena sílice y diferentes porcentajes de fibra de Luffa $(0.3,0.6$ y $0.9 \%)$. Para iniciar la polimerización de la resina, se necesita agregar entre 1 y $2 \%$ en peso de peróxido de metil etil cetona por cada $100 \mathrm{~g}$ de resina.

\section{Irradiación de los concretos y evaluación de las propiedades mecánicas}

Los concretos poliméricos fueron irradiados con rayos gamma a 50 y 100 kGy en un irradiador industrial JS6500 , que cuenta con lápices de cobalto $60\left({ }^{60} \mathrm{Co}\right)$ de vida media de 5.2 años. Irradiador localizado en el Instituto Nacional de Investigaciones Nucleares de México (ININ).

La evaluación de la resistencia mecánica a la compresión y a la flexión de las probetas de concreto polimérico se realizó en una Maquina Universal de Pruebas marca Controls ${ }^{\mathrm{MR}}$ con capacidad de 30 toneladas. Mientras que el módulo de elasticidad dinámico con un equipo de ultrasonido modelo 58-E0048 (Controls $^{\text {TM }}$, Cernusco, Italy), ambos equipos localizados en el Laboratorio de Investigación y Desarrollo de Materiales Avanzados (LIDMA), de la Facultad de Química de la Universidad Autónoma del Estado de México.

\section{Microscopía Electrónica de Barrido}

Una vez efectuado las pruebas mecánicas a los concretos, se analizaron regiones de las fibras de luffa en un microscopio electrónico de barrido, marca JEOL modelo JSM-6510LV a un voltaje de $20 \mathrm{kV}$, el cual se encuentra localizado en el Centro de Investigación en Química Sustentable (CIQS) de la Universidad Autónoma del Estado de México. El equipo tiene una resolución de $3.0 \mathrm{~nm}$ y voltajes de aceleración de 0.5 a $30 \mathrm{kV}$.

\section{RESULTADOS}

\section{Resistencia a la compresión}

El concreto polimérico tomado como control (sin fibras y sin irradiación), presenta una resistencia a la compresión de $27 \mathrm{MPa}$ (Figura 1); la cual se incrementa hasta un máximo de $27 \%$ cuando se irradia a 100 kGy. Este incremento puede atribuirse a la modificación estructural de la resina de poliéster insaturado después de ser irradiada; es decir la energía ionizante produce la formación de enlaces entre cadenas poliméricas de la resina (cross-linking), incremento en el grado de polimerización y reticulado de la matriz; dejando a las partículas de arena sílice atrapadas en un red tridimensional formada por la resina irradiada. 
Para los concretos poliméricos con fibras, se observa que la resistencia a la compresión disminuye gradualmente al incrementar el porcentaje de fibra de luffa; la máxima reducción fue de $30 \%$, obtenida para concretos con $0.9 \%$ de fibras, respecto al concreto control. Tal disminución se puede atribuir a varias situaciones: a) La alta porosidad de las fibras de Luffa; en general los poros actúan como fallas en el composito durante el esfuerzo de compresión, por lo que la resistencia disminuye; b) al débil enlace fibramatriz, generando regiones con separación de ambos componentes; c) la naturaleza opuesta de los materiales, por un lado la fibra de Luffa es hidrofílica y por otro la resina poliéster es hidrofóbica.

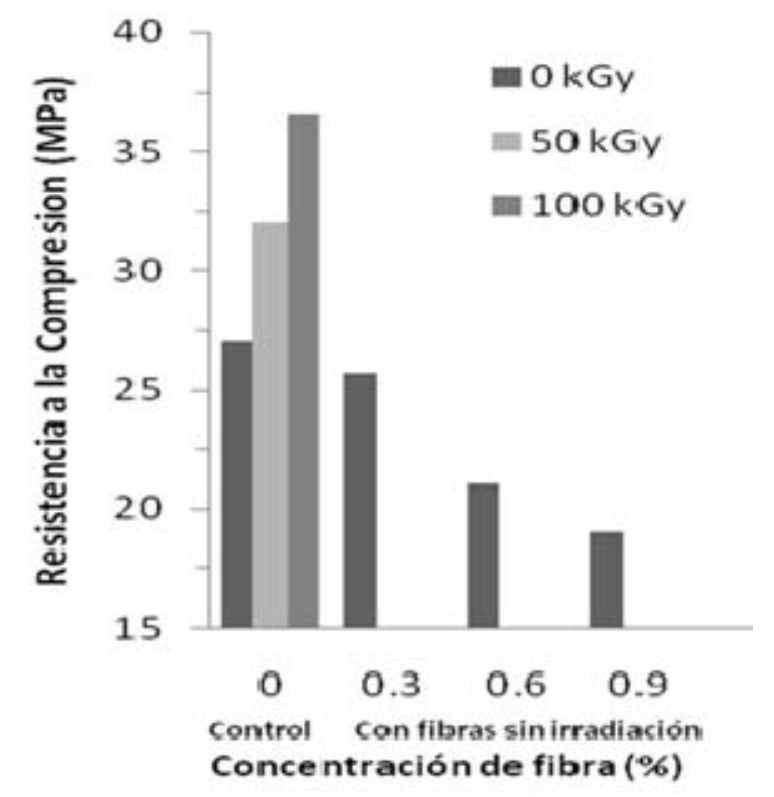

Fig. 1: Resistencia a la compresión de concretos poliméricos.

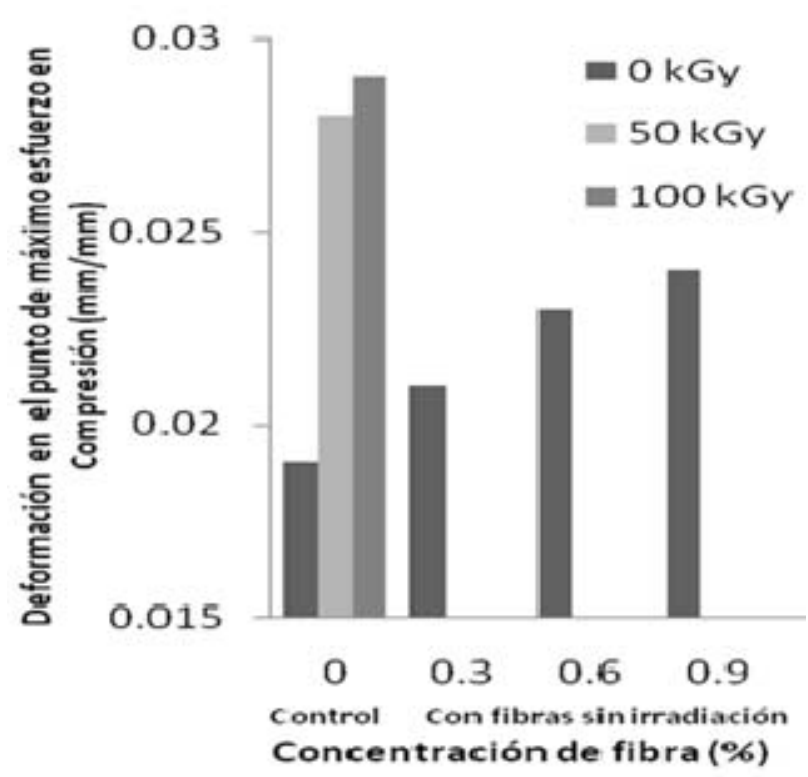

Fig. 2: Deformación en el punto de máximo esfuerzo en compresión de concretos poliméricos

La Figura 2 muestra la deformación de los concretos poliméricos durante el ensayo de compresión. Se observa que la deformación del concreto polimérico sin fibras es de $0.019 \mathrm{~mm} / \mathrm{mm}$, la es un poco más de tres veces mayor que la deformación estándar para concretos poliméricos, reportada en la literatura de $0.006 \mathrm{~mm} / \mathrm{mm}$. Al aplicar radiación se incrementa el valor de la deformación hasta un máximo de 34\% cuando se irradia a $100 \mathrm{kGy}$. Un comportamiento similar se observa para los concretos con fibras, es decir los valores se incrementan a medida que aumenta la concentración de fibras. El máximo porcentaje de aumento es de $20 \%$ (concreto con $0.9 \%$ de fibra), respecto del concreto tomado como control. Tal aumento se debe a la participación de las fibras en la absorción de los esfuerzos de compresión aplicado. Las fibras añadidas producen un concreto con mayor deformación plástica.

\section{Resistencia a la flexión}

La Figura 3 muestra la resistencia a la flexión de los concreto poliméricos. Se observa dos comportamientos: a) Para el concreto tomado como control, el valor sin irradiación es de $10 \mathrm{MPa}$, el cual disminuye hasta $6 \%$ cuando se irradia a 50 y 100 kGy; a diferencia de la resistencia a la compresión la cual aumenta cuando la dosis de radiación aumenta (ver Figura 1); b) Para concretos con fibras, la resistencia a la flexión disminuye a medida que se incrementa la concentración de fibras (El mismo comportamiento se observó para la resistencia a la compresión). Esta disminución se asocia a la fibras de luffa, por un lado su alta porosidad (los poros actúan como fallas en el concreto) y por otro al grado de humedad (absorción de líquido); los cuales afectan la unión física entre la resina de poliéster y las fibras.

En la Figura 4 se muestra la deformación debida al esfuerzo de flexión de concretos poliméricos. Se observa: a) Para el concreto control una deformación en el punto de máximo esfuerzo de $0.41 \mathrm{~mm}$, la cual aumenta conforme aumenta la dosis de radiación, hasta en un 8\% cuando se irradia a 100 kGy; b) Para concretos con fibras la deformación se incrementa gradualmente conforme se incrementa la concentración de fibra. El mayor incremento se obtiene con $0.9 \%$ de fibra y representa un $28 \%$ de aumento. 


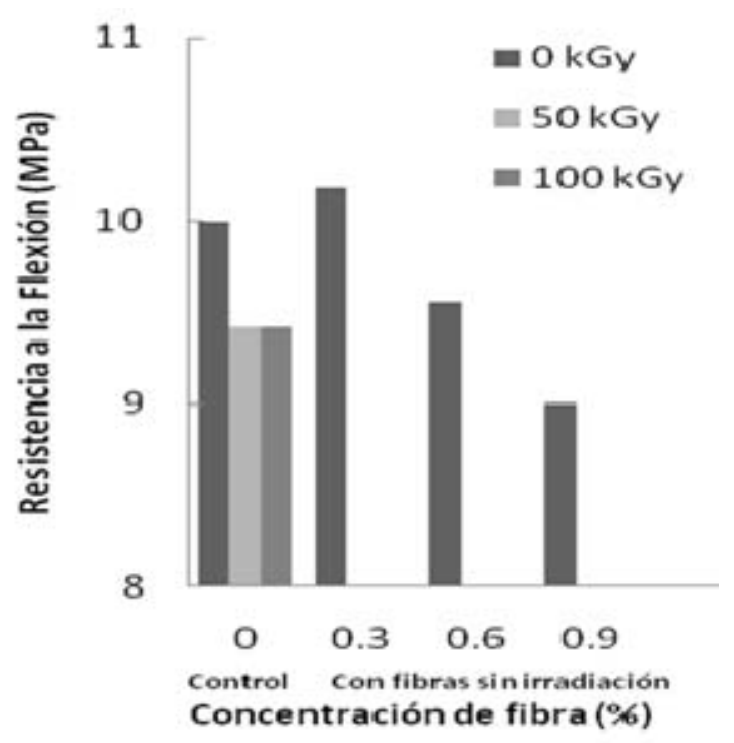

Fig. 3: Resistencia a la flexión de concretos poliméricos

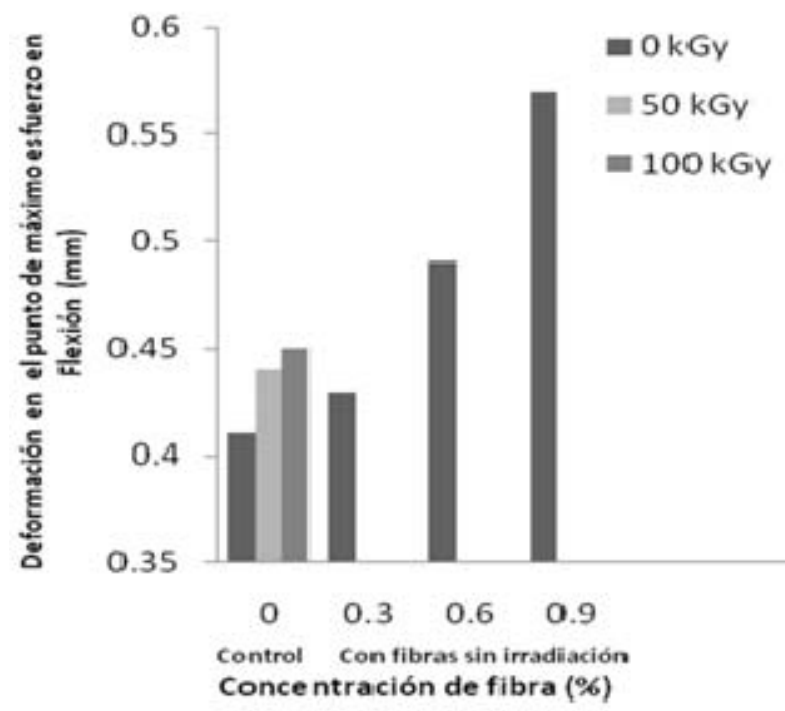

Fig. 4: Deformación en el punto de máximo esfuerzo en flexión de concretos poliméricos.

\section{Módulo de Young y Módulo de elasticidad dinámico}

La Figura 5 muestra los valores obtenidos para el módulo de elasticidad de concretos poliméricos. Se observa: a) Para el concreto control un módulo de elasticidad de $2.8 \mathrm{GPa}$, el cual disminuye cuando se irradian los concretos; llegando a ser hasta 32\% menor cuando se irradia a $50 \mathrm{kGy}$; b) Para los concretos con fibras se observa la disminución en los valores al incrementar el porcentaje de fibra. Estos valores son hasta $32 \%$ menores (concreto con $0.9 \%$ de fibra), respecto al concreto control. Con base en la definición del módulo de Young, al disminuir los valores se está obteniendo en forma gradual un material con mayor grado de elasticidad (dúctil), lo cual es provocado por la presencia de las fibras de luffa en el concreto.

Los módulos de elasticidad dinámica se muestran en la Figura 6. Se observa: a) Para concreto control un módulo de elasticidad dinámico de $5.2 \mathrm{GPa}$, el cual se va incrementando conforme se incrementa la dosis de radiación, hasta un 16\% mayor para una dosis aplicada de 100 kGy; b) Para los concretos con fibras se observa disminución en los valores al incrementar la concentración de fibra. Estos valores son hasta $23 \%$ menores (concreto con $0.9 \%$ de fibra), respecto al concreto control. El mismo comportamiento fue observado para el módulo de elasticidad medido por compresión, observado en la Figura 5.

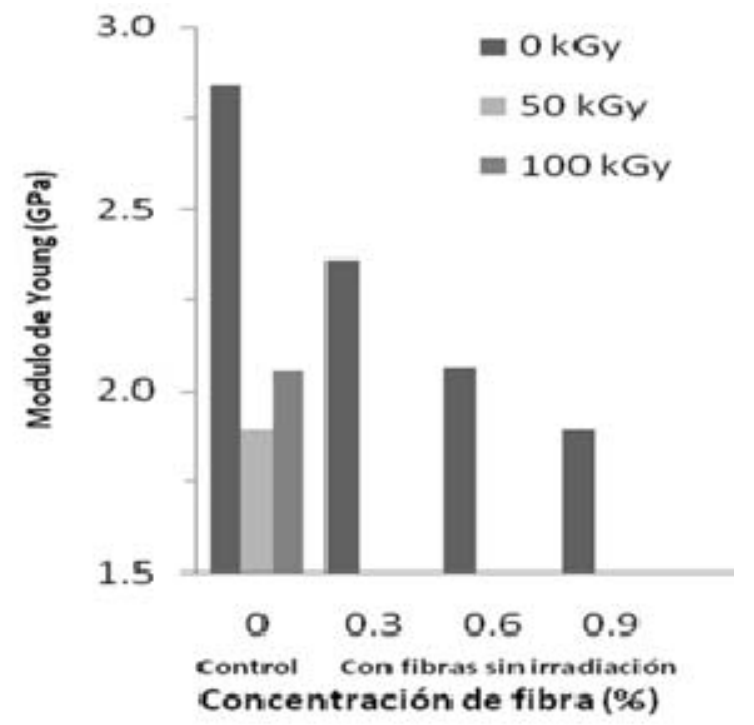

Fig. 5: Módulo de Young de concretos poliméricos

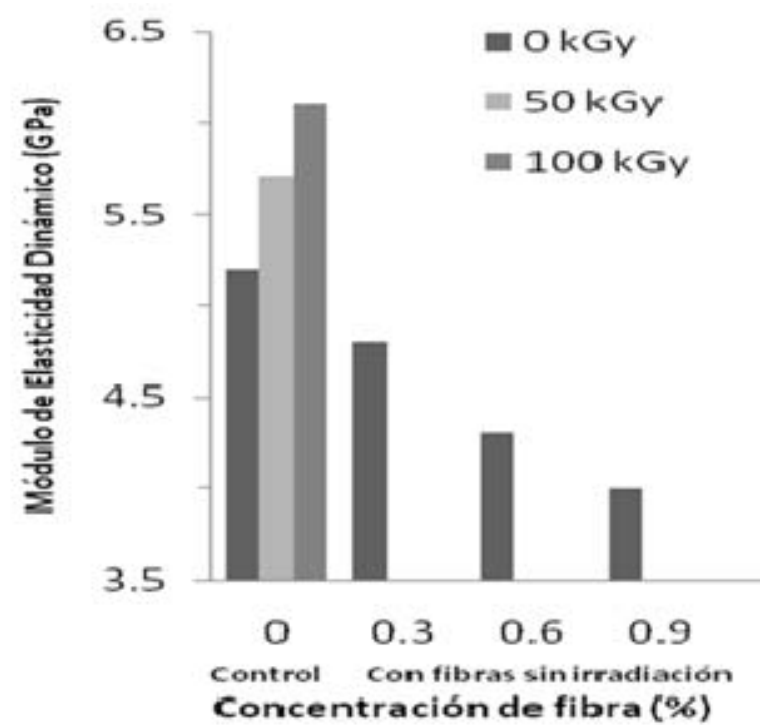

Fig. 6: Módulo de elasticidad dinámico de concretos poliméricos. 
El comportamiento mecánico de la disminución en la resistencia a la compresión y a la flexión, y en los módulos de elasticidad; así como el aumento en las deformaciones en el punto de máximo esfuerzo, se puede relacionar con las imágenes de microscopia electrónica de barrido de las fibras de luffa.

En la Figura 7a se observan los poros en el interior de las fibras. Estos poros actúan como fallas en el concreto durante el ensayo de compresión, lo que disminuye la resistencia. Mientras que en la Figura $7 \mathrm{~b}$ se observa una superficie rugosa con partículas de lignina con diferentes formas y la presencia de microcanales con anchuras que van de 4 a $12 \mu \mathrm{m}$. Algunos de estos micro-canales de la superficie de la fibra no pueden ser llenados por completo por arena sílice o resina poliéster durante el proceso de elaboración del concreto, por lo que se genera cierto grado de porosidad en el interior del concreto. Finalmente, en la Figura 7c se observa la presencia de una delgada capa de lignina y hemi-celulosa que cubre las fibrillas celulósicas. Se ha reportado la presencia de mayor numero de partículas de lignina cuando las fibras de luffa se irradian a altas dosis de radiación gamma (Ghali L. et al., 2011). Aparte del efecto que produce el grado de porosidad, se debe añadir el que produce la humedad en las fibras, la cual produce un débil enlace entre la resina poliéster y la fibra, y por lo tanto una deficiente transferencia de esfuerzos.
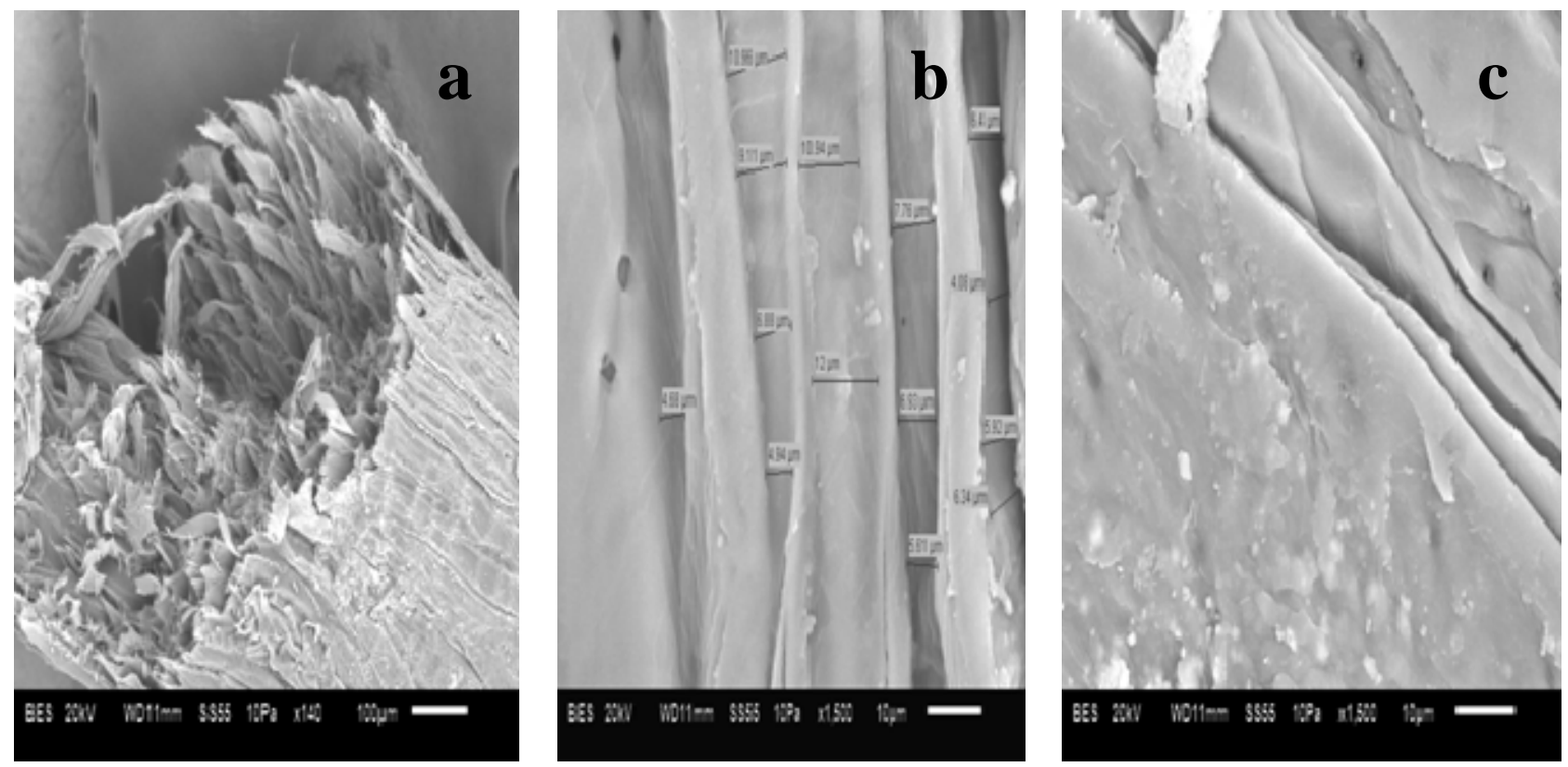

Fig. 7: Imágenes de SEM de fibras de luffa a diferentes aumentos: a) x140, b) x1500 y c) x1500.

\section{CONCLUSIONES}

Con base en los resultados se concluye que las propiedades mecánicas dependen tanto de la concentración de fibra de luffa como de la dosis de radiación aplicada. Para concretos sin fibras e irradiados se encontró aumento en la resistencia a la compresión y en la deformación en el punto de máximo esfuerzo, pero disminución de la resistencia a la flexión y del módulo de Young, esto pone de manifiesto que la polimerización de la resina poliéster mediante radiación gamma está generando un concreto polimérico con mayor grado de resistencia mecánica pero al mismo tiempo más dúctil, lo cual es difícil de lograr en un concreto polimérico convencional, y mucho menos en un concreto hidráulico (a base de cemento Portland). Por otro lado, para concretos con fibras disminuyen la resistencia a la compresión, a la flexión y los módulos de elasticidad; sin embargo aumenta la deformación en el punto de máximo esfuerzo; esto indica que al ir agregando fibras de luffa se va generando un concreto polimérico menos resistente pero con mayor grado de elasticidad.

\section{REFERENCIAS}

Altinisik A., E. Gur y Y. Seki, A natural sorbent, Luffa cilíndrica for the removal of a model basic dye, Journal of Hazardous Materials 179, 658-664 (2010).

Boynard C.A. y J.R.M. D'almeida, Morphological characterization and mechanical behavior of sponge gourd (luffa cylindrica)-polyester composite materials, Polymer-Plastics Technology and Engineering 39 (3), 489 499 (2010). 
Clough R.L. High-energy radiation and polymers: A review of commercial processes and emerging applications Nucl. Instr. Meth. Phys. Res. B 185, 8-33 (2001).

Cruz-Zaragoza E. y G. Martínez-Barrera: Ionizing radiation effects on the matter and its applications in research and industry. In 'Gamma radiation effects on polymeric materials and its applications' (eds.: Barrera-Díaz C., Martínez-Barrera G.), Research Signpost, Kerala India, 1 - 14 (2009).

Ghali L., S. Msahli, M. Zidi y F. Sakli, Effect of pre-treatment of Luffa fibres on the structural properties, Materials Letters 63(5), 61-63 (2009)

Ghali L., M. Aloui, M. Zidi, H. Bendaly, S. Msahli y F. Sakli, Effect of chemical modification of luffa cylindrica fibers on the mechanical and hygrothermal behaviours of polyester/luffa composites, BioResources 6, 38363849 (2011).

Kunst S., J.F. Matos, M.A. Korb y C.F Malfatti. Obtención y Caracterización de Revestimientos Protectores a Base de Silanos para la Protección de Aceros Galvanizados. Inf. Tecnol. 22 (5), $67-76$ (2011).

Martínez-Barrera G., Uso de fibras poliméricas en el mejoramiento de las propiedades mecánicas del concreto, en "Tópicos en Ciencia de Materiales", Universidad Autónoma del Estado de México, México, 125 -170 (2009).

Martínez-Barrera G. y W. Brostow, Fiber-reinforced polymer concrete: Property improvement by gamma irradiation. In 'Gamma radiation effects on polymeric materials and its applications' (eds.: Barrera-Díaz C., Martínez-Barrera G.), Research Signpost, Kerala India, 27 - 44 (2009).

Martínez-Barrera G., L.F. Giraldo, B.L. López y W. Brostow, Effects of gamma radiation on fiber-reinforced polymer concrete, Polym. Compos. 29 ,1244-1251 (2008).

Mehdi A., Structural Reinforcement of Building Materials using Polymer Concrete American Journal of Scientific Research 23, 135-143 (2011).

Miro C., J.M. Avila, M. García y J. Pastor-Villegas, Riesgos Debido a la Radiactividad Natural de Pizarras de Construcción. Inf. Tecnol. 21 (1), 9-16 (2010).

Oboh I.O. y E.O. Aluyor, Luffa cylindrica - an emerging cash crop, African Journal of Agricultural Research 4 (8), 684-688 (2009).

Pistor V., S.S.S dos Santos Soares, H.L. Ornaghi Júnior, R. Fiorio y A.J. Zattera, Influence of Glass and Sisal Fibers on the Cure Kinetics of Unsaturated Polyester Resin, Materials Research 15 (4), 1 - 7 (2012).

Reis J.M.L. y E.P. Carneiro, Effect of piassava lees in the fracture behavior of polymer mortars, Composite Structures 95, 564-568 (2013)

Santos Delgado P., S.L. Bragança Lana, E. Ayresa, P.O. Santiago Patrício y R. Lambert Oréficec, The Potential of Bamboo in the Design of Polymer Composites, Materials Research 15 (4), 1 - 6 (2012).

Tanobe V.O.A., T.H.D. Sydenstricker, M. Murano y S.C. Amico, A comprehensive characterization of chemically treated Brazilian sponge-gourds (Luffa cylindrica), Polymer Testing 24 (4), 474-482 (2005).

Valcuende M., J. Benlloch y C. Parra, Estudio Experimental de Piezas Lineales de Hormigón Reforzadas con Fibras de Carbono. Inf. Tecnol. 15 (6), 23-28 (2004).

Zollo R.F., Fiber-reinforced concrete: an overview after 30 years of development, Cement and Concrete 19, 107-122 (1997). 
